\title{
UN MÉTODO EMPÍRICO PARA LA SELECCIÓN DE MODELOS DE GESTIÓN DE TESORERÍA
}

\section{AN EMPIRICAL METHOD TO SELECT CASH MANAGEMENT MODELS}

Francisco Salas-Molina ${ }^{1}$

1. Ingeniero de Organización Industrial y Master en Creación de Empresas de Base Tecnológica. Director de I+D y Sistemas de Información en Hilaturas Ferre, S.A., Banyeres de Mariola, Alicante, (España). E-Mail: francisco.salas.molina@gmail.com

Citación sugerida:

Salas-Molina, F. (2017). Un método empírico para la selección de modelos de gestión de tesorería. $3 C$ Tecnología: glosas de innovación aplicadas a la pyme, 6(3). 1-13. DOI: $<$ http://dx.doi.org/10.17993/3ctecno.2017.v6n3e23.1-13/>. 


\section{RESUMEN}

La creciente posibilidad de acceder a grandes bases de datos transaccionales ha potenciado enormemente las finanzas corporativas empíricas. En este trabajo, proponemos explotar la dimensión empírica de la gestión de tesorería. Para ello, aplicaremos técnicas de (big) data sobre flujos de caja empíricos que son cruciales para la selección y ajuste de modelos de gestión de tesorería.

\section{ABSTRACT}

The increasing possibility to access big transactional data bases has boosted empirical corporate finance. In this work, we propose to exploit the empirical dimension of cash management. To this end, we apply (big) data techniques to empirical cash flows that are crucial to the selection and setting of cash management models.

\section{PALABRAS CLAVE}

Big data, finanzas, gestión de tesorería, previsión, control.

\section{KEY WORDS}

Big data, finance, cash management, forecasting, control. 


\section{INTRODUCCIÓN}

La gestión de tesorería se ocupa de la planificación y el control de los recursos financieros de la empresa a corto plazo. Desde un punto de vista cuantitativo, el objetivo básico es decidir qué cantidad de recursos se mantienen en caja por motivos operacionales, de precaución o incluso de especulación (Ross, 2002). Implícitamente, esta decisión supone invertir a corto plazo los recursos excedentes para obtener un ingreso financiero.

La investigación sobre la gestión de tesorería ha seguido un camino central caracterizado por modelos de gestión de tesorería basados en límites de control a partir del modelo de Miller (1966). Desde los años 60 del pasado siglo, los investigadores han propuesto un buen número de modelos alternativos que trataban de resolver alguna limitación de los anteriores o de considerar algún aspecto que no había sido tenido en cuenta (da Costa Moraes, 2015).

Desde un punto de vista práctico, el tesorero de una empresa se enfrenta a tarea cuya resolución no es en modo alguno trivial: decidir qué modelo debe aplicar su empresa y por qué razón. Comprender la metodología que hay detrás de decenas de propuestas y, sobre todo, verificar si se cumplen las asunciones de partida de estas propuestas en cada caso concreto es una tarea cuanto menos compleja.

Las negativas consecuencias que se podrían derivar de tomar decisiones sin un completo análisis de cada situación, justifican la dedicación de los esfuerzos necesarios para mejorar la gestión de una disciplina financiera habitualmente poco automatizada. De hecho, cualquier empresa con beneficios puede cesar su actividad si no es capaz de mantener un nivel mínimo de tesorería para sus operaciones habituales.

En la actualidad, uno de los recursos al alcance de cualquier tesorero es la gran cantidad de datos que continuamente se va generando en su empresa. Desgraciadamente, la dimensión empírica de las finanzas no se ha desarrollado a la misma velocidad que su dimensión teórica. Esta circunstancia representa un obstáculo a la hora de trasladar a la práctica diaria los resultados de la investigación en el ámbito de las finanzas.

Por todo ello, este trabajo pretende mejorar la toma de decisiones basadas en datos en el ámbito de la gestión de tesorería basado en tres aspectos básicos como se muestra en el Gráfico 2. En primer lugar, los datos constituyen la materia prima fundamental con la que ajustar y validar un modelo de gestión de tesorería. El estudio empírico de los datos facilita la comprensión de los modelos más avanzados de gestión de tesorería. Finalmente, estos modelos permiten una toma de decisiones más eficiente $y$, hasta cierto punto, automatizada 


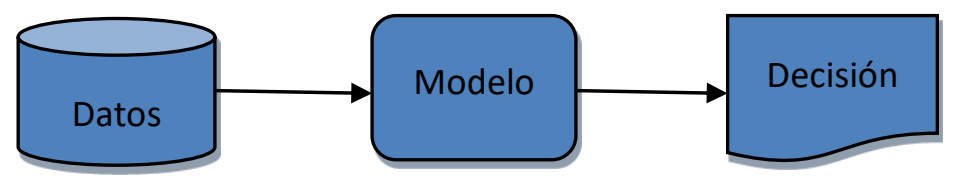

Gráfico 1. Decisiones basadas en datos.

Fuente: elaboración propia.

A continuación, tras analizar las principales dimensiones del problema de gestión de tesorería, se ofrecen directrices para el análisis empírico de los flujos de caja de una empresa. Las conclusiones de este análisis son cruciales para la selección de alguno de los modelos propuestos en la literatura. Adicionalmente, se propone un método para la validación empírica del modelo a partir de técnicas de (big) data. Como resultado final, se describe un método para seleccionar el modelo de gestión de tesorería más adecuado para una empresa a partir de datos empíricos.

\section{SELECCIÓN EMPÍRICA DE MODELOS DE GESTIÓN DE TESORERÍA}

La gestión de tesorería tiene como objetivo principal hacer un uso eficiente de los recursos financieros a corto plazo de una empresa. Para ello, el enfoque habitual propuesto en la literatura plantea el problema de gestión de tesorería desde una perspectiva de control. Básicamente, el tesorero dispone en cada momento de cierto nivel de efectivo cuyo nivel tiene que controlar para mantenerlo entre ciertos niveles que se consideran adecuados para evitar tanto saldos negativos como saldos excesivos que podrían estar generando un determinado ingreso financiero.

A continuación analizamos las principales dimensiones del problema de gestión de tesorería como marco de trabajo para nuestra posterior propuesta metodológica.

\subsection{DIMENSIONES DEL PROBLEMA DE GESTIÓN DE TESORERÍA}

De la literatura sobre gestión de tesorería es posible extraer una serie de aspectos o dimensiones del problema de gestión de tesorería que habitualmente son analizados para conseguir una mejor comprensión del problema. Estas dimensiones se recogen en el Gráfico 2. 


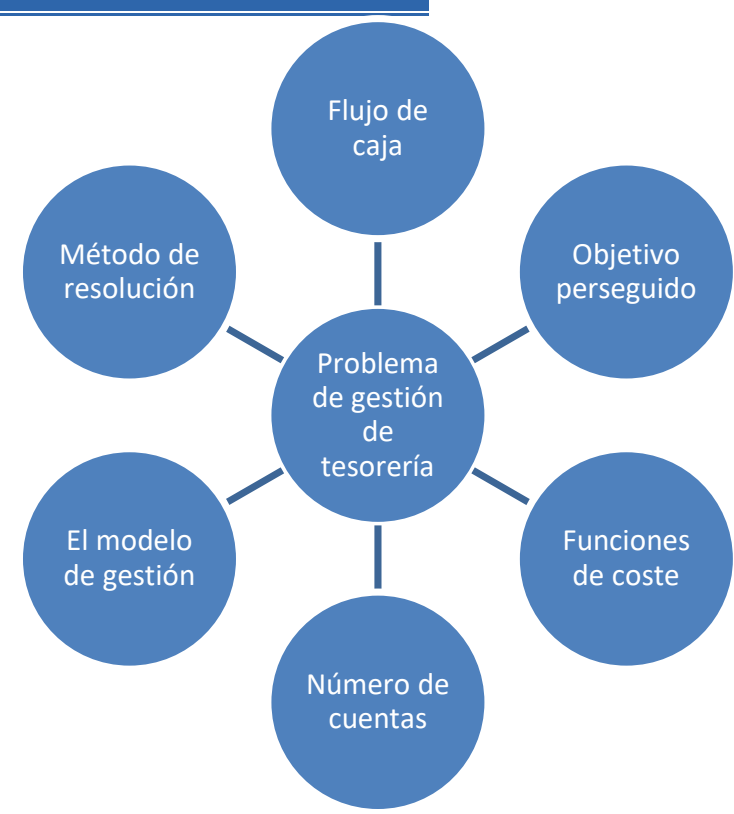

Gráfico 2. Las dimensiones del problema de gestión de tesorería.

Fuente: elaboración propia.

Aunque no es necesario seguir un orden estricto para abordar las diferentes dimensiones del problema de gestión de tesorería, sí que conviene seguir cierto orden lógico ya que las decisiones que se tomen en una dimensión pueden afectar al resto de dimensiones. Debido a su importancia, en este trabajo proponemos que el análisis comience en el flujo de caja y se desarrolle en sentido horario según el Gráfico 2.

El flujo de caja y sus principales propiedades estadísticas constituyen la dimensión clave de la gestión de tesorería. De hecho, la propuesta metodológica que se describe en este trabajo está basada en el análisis empírico de los flujos de caja. Sin embargo, a pesar de que la hipótesis más extendida en la literatura acerca de la distribución estadística de los flujos de caja es la de normalidad, hay pocas evidencias empíricas en este sentido sino más bien al contrario (Pindado, 1996). Esta circunstancia marca necesariamente la selección del modelo tal como se argumenta a continuación.

El objetivo perseguido por el decisor es principalmente la minimización de costes. Sin embargo, recientemente están apareciendo nuevas propuestas de optimización multiobjetivo en las que tanto el coste como el riesgo son tenidos en cuenta para obtener las mejores estrategias (Salas-Molina et al., 2016).

La definición de las funciones de coste que formarán parte de la función objetivo a optimizar es la siguiente dimensión del problema de gestión de tesorería. Tal como se muestra en el Gráfico 3, los costes considerados habitualmente en la gestión de tesorería son los de mantenimiento (por tener un saldo de caja improductivo) y los de transacción (por transferir parte del saldo a determinadas inversiones financieras temporales). 


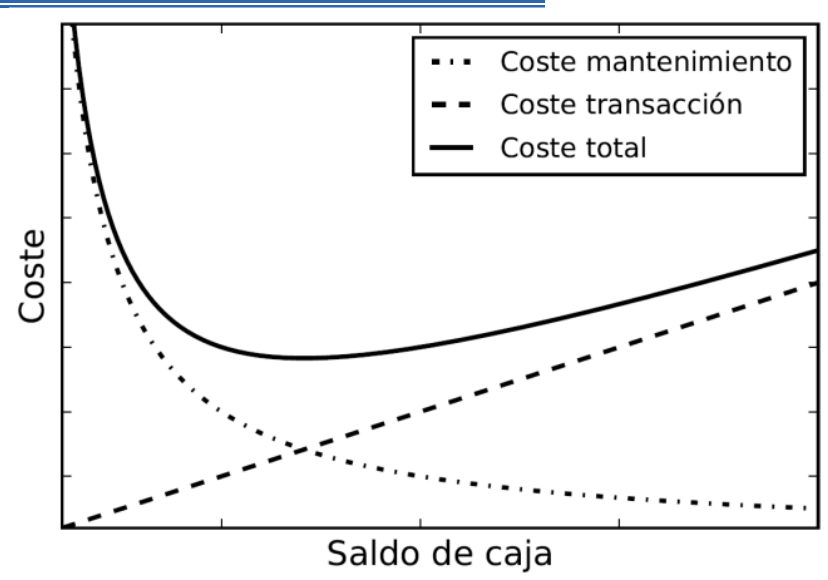

Gráfico 3. Costes de la gestión de tesorería. Fuente: elaboración propia.

Por otro lado, el marco de análisis habitual en la gestión de tesorería contempla dos tipos de activos: una cuenta de efectivo y una cuenta de inversión. En ambos casos, estas cuentas agregan los saldos disponibles en las posibles distintas cuentas de efectivo y de inversión con las que se trabaje.

Otro de los aspectos críticos en la gestión de tesorería es el modelo utilizado para definir las acciones de control necesarias para mantener el saldo entre los niveles deseados al tiempo que se minimizan las funciones de coste establecidas. En este caso, el abanico de disponibilidad es lo suficientemente grande como para plantear ciertas dudas que tratamos de resolver en este trabajo. Uno de los más relevantes es el modelo de Miller y Orr (1966) que está basado en tres niveles de manera que cuando el saldo alcanza el nivel superior ( $h$ ) o el inferior (I), la acción de control lleva el saldo de nuevo al nivel medio (z), tal como se muestra en el Gráfico 4.

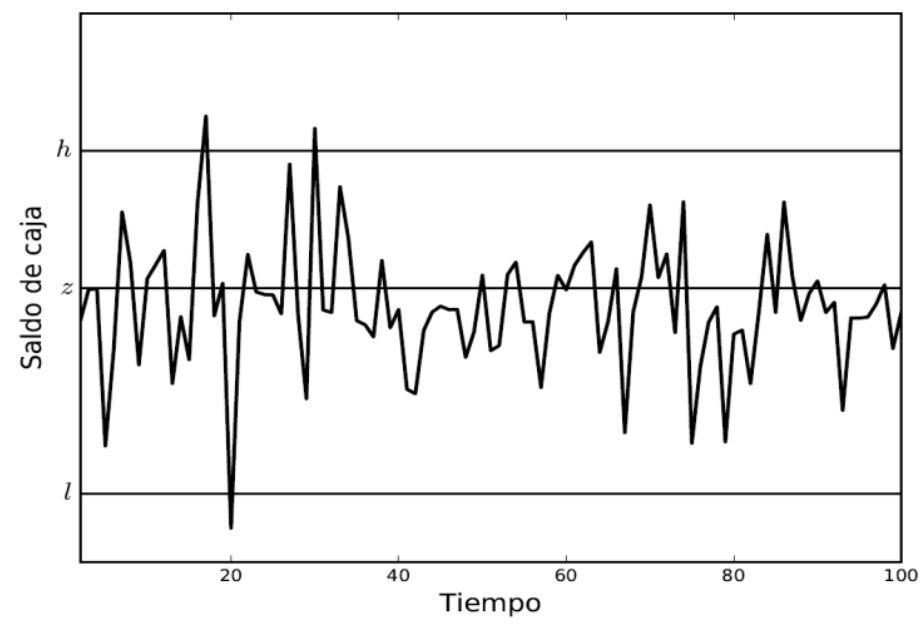

Gráfico 4. El modelo de Miller y Orr.

Fuente: elaboración propia. 
La última de las dimensiones está íntimamente relacionada con la selección del modelo gestión de tesorería. No solamente hay que seleccionar un modelo sino también hay que ajustar los parámetros de control inherentes al modelo. Por ejemplo, en el modelo de MillerOrr habría que determinar los parámetros $h, z$, y I. En este aspecto, también existe una gran variedad de métodos de resolución con complejidad variable desde la aplicación de una fórmula matemática (Miller y Orr, 1966) a la utilización de algoritmos genéticos (Gormley y Meade, 2007).

Una vez que ya conocemos las principales dimensiones de la gestión de tesorería, podemos plantearnos cómo un análisis empírico puede despejar algunas de las dudas a la hora seleccionar un modelo de gestión.

\subsection{ANÁLISIS EMPÍRICO DE LOS FLUJOS DE CAJA}

La primera cuestión que debemos hacernos al abordar el problema de gestión de tesorería es con qué materia prima vamos a trabajar o, en otras palabras, con qué tipo de flujos de caja contamos. No todos los flujos de caja son iguales. Los hay mayores y menores, ciertos e inciertos. Los flujos de caja mayores son todos aquellos de los que se conoce con exactitud tanto el importe como la fecha en la que se producirán. Algunos ejemplos son los vencimientos de préstamos, los pagos de impuestos, nóminas o dividendos. Los flujos de caja menores son todos aquellos sobre los que existe cierta incertidumbre tanto sobre el importe como en la fecha en que se producirán. El ejemplo más claro de flujo de caja menor son los cobros de clientes. Aunque exista un importe y una fecha de pago acordada, es bastante habitual que ni el primero ni la segunda acaben cumpliéndose.

La separación de los flujos de caja por tipo puede hacerse aún más detallada si somos capaces de identificar cuáles son sus componentes más importantes. Los componentes más evidentes del flujo de caja son los cobros y los pagos, pero podemos ir un poco más allá y realizar una separación por naturaleza, por origen o por destino, o por cualquier otro criterio. Por ejemplo, pagos a proveedores de materia prima, pagos a proveedores de financiación, pagos a proveedores de servicios de electricidad, de agua, de gas, de material de oficina. La separación puede ser todo lo minuciosa que nos interese. Esta separación puede resultar de gran utilidad debido a que es probable cada componente tenga unas características diferentes.

De la división de flujos de caja entre mayores o menores se deriva una primera consecuencia importante. Los flujos de caja mayores se conocen con exactitud y, por tanto, son fácilmente predecibles. Diremos de ellos que forman la parte determinista de nuestros flujos de caja totales. La parte restante, que llamaremos estocástica, merece un análisis estadístico en profundidad.

Las asunciones más comunes en la literatura sobre las propiedades estadísticas suponen que los flujos de caja son: 
- Normales: cuando las observaciones se distribuyen simétricamente alrededor de un valor medio y con una varianza finita según la curva de Gauss.

- Independientes: cuando las observaciones actuales no están correlacionadas con observaciones pasadas.

- Estacionarios: cuando la media y la varianza de las observaciones no varían con el tiempo.

Sin embargo, existe poca evidencia empírica sobre el cumplimiento de estas condiciones cuando se analizan datos reales. La forma más habitual de comprobar el cumplimiento de estas condiciones es mediante la utilización de un test estadístico. Por ejemplo, el test de Kolmogorov-Smirnov para normalidad o el test de Ljung-Box test para independencia, o el test de Dickey-Fuller para estacionariedad. Otros métodos más intuitivos son el cálculo de los coeficientes de asimetría y curtosis para comprobar la normalidad, los mapas de Poincaré para independencia, o los gráficos de medias y varianzas móviles para estacionariedad.

En este punto, es importante recordar que calificar de estocásticos los flujos de caja no significa que sean impredecibles. De hecho, la estacionalidad y la existencia de posibles patrones ocultos en los datos es una cuestión ampliamente estudiada y que puede ser utilizada en beneficio de la reducción de costes (Salas-Molina et al., 2017). En este sentido, la aplicación de técnicas de (big) data para la previsión de los flujos de caja a partir de un conjunto de variables explicativas constituye un elemento crucial en la selección empírica del modelo de gestión de tesorería más adecuado para cada empresa.

\subsection{SELECCIÓN DEL MODELO BASADA EN DATOS}

Para abordar la selección de modelos de gestión de tesorería consideremos la siguiente situación que puede darse con frecuencia. Un tesorero desea empezar a controlar el saldo de sus cuentas mediante un modelo de gestión de tesorería. A priori, un modelo sencillo con tres niveles de control como el de Miller y Orr (1966) representado en el Gráfico 3, no le parece complicado de implementar. Para determinar estos niveles de control, nuestro tesorero descubre con alivio que simplemente tiene que aplicar unas fórmulas sencillas que dependen de la desviación típica de los flujos de caja y de los costes de mantenimiento y transacción. Finalmente se da cuenta de que estas fórmulas sólo son óptimas cuando los flujos de caja son normales, independientes y estacionarios (NIE). Tras realizar un análisis empírico de sus datos de flujo de caja tal como se describe en la sección anterior, los resultados muestran que estas condiciones no se cumplen.

Del análisis de este tipo de situaciones se derivan dos cuestiones centrales para la gestión de tesorería:

1. ¿Qué modelo elegir? (Dimensión modelo)

2. ¿Cómo ajustar y validar el modelo elegido? (Dimensión método de resolución) 
En esta sección abordaremos la primera de estas cuestiones y dejaremos la segunda para la siguiente sección. Para la selección inicial del modelo, nos basaremos principalmente en los resultados del análisis empírico: primero, en las hipótesis NIE; segundo, en la posibilidad de predecir los flujos de caja. Por tanto, se asume que disponemos de un conjunto de datos de flujos de caja históricos sobre los que trabajar.

Supongamos que tras el estudio empírico, se cumplen las NIE. En este caso, planteamos (por simplicidad) dos opciones: el modelo de Miller y Orr (1966) con tres niveles cuando los costes variables de transacción son nulos, y el modelo de Eppen y Fama (1968) con cuatro niveles cuando se soportan costes de transacción fijos y variables.

En el caso de que alguna de las NIE no se cumpla, es posible plantear alguna técnica de transformación de datos como la de Box-Cox para conseguir normalidad, o el cálculo de diferencias entre períodos consecutivos para conseguir independencia. Si aun así, no es posible cumplir las NIE podemos considerar modelos alternativos.

Supongamos ahora que tras aplicar alguna de las técnicas de previsión de series temporales (Salas-Molina et al., 2017), somos capaces de predecir con cierta precisión los flujos de caja que se van producir en los próximos días. En este caso planteamos dos nuevos modelos: el modelo de Stone (1972) basado en cinco niveles de control; y el modelo de Gormley y Meade (2007) basado en cuatro niveles. Ambos modelos utilizan previsiones de los flujos de caja futuros para decidir las acciones de control a establecer. El modelo de Stone acumula las previsiones de los próximos períodos con el objetivo de reducir el número de transacciones mientras que el modelo de Gormley y Meade plantea una acción de control para cada período de tiempo. En este caso, no hay una razón clara para decantarse por uno u otro. La selección definitiva dependerá en última instancia de un aspecto adicional que ya hemos apuntado: el ajuste y la validación del modelo.

\subsection{AJUSTE Y VALIDACIÓN DEL MODELO}

Por ajuste del modelo, nos referimos a la selección de los parámetros o niveles de control necesarios para realizar las transacciones oportunas. Por validación del modelo, nos referimos a algún procedimiento basado en datos que permita comparar el modelo con otros modelos alternativos o incluso con una estrategia trivial, por ejemplo, si no aplicáramos ninguna acción de control.

Para la determinación de los niveles de control, los autores de los modelos propuestos propusieron distintos métodos. Mientras que Eppen y Fama utilizó programación dinámica, Stone simplemente sugería la simulación cómo método para calcular los niveles. Más recientemente, Gormley y Meade recomienda el uso de algoritmos genéticos. La gran variedad de métodos propuestos hacen del ajuste del modelo una tarea complicada cuando se barajan distintas opciones.

Con el objetivo de resolver esta limitación, en este trabajo proponemos realizar el ajuste de cualquier modelo basado en niveles mediante simulación Monte Carlo tal como se describe 
en Salas-Molina et al. (2016). Las técnicas de simulación Monte Carlo se basan en la realización de un número elevado de experimentos que posteriormente son evaluados de acuerdo con una función objetivo dada. La ley de los grandes números asegura que las estimaciones derivadas de este análisis convergen a los valores reales a medida que el número de experimentos aumenta. Este tipo de técnicas son especialmente útiles cuando existe un número finito de posibles combinaciones a evaluar como es el caso de la selección de niveles de control discretos entre un valor mínimo y un valor máximo.

El paso final de nuestra propuesta de selección de modelos de gestión de tesorería, es una validación empírica basada en un concepto ampliamente utilizado en el ámbito del (big) data: la validación cruzada. La idea básica de la validación cruzada es separar el conjunto de datos disponibles en dos partes: la primera parte se utilizará para ajustar el modelo (training set); y la segunda se utilizará para validar el modelo (test set). Por ejemplo, podemos utilizar el $80 \%$ de los datos de flujo de caja más antiguos para calcular los mejores límites de control del modelo. Posteriormente, utilizaremos el $20 \%$ restante para evaluar el comportamiento del modelo con datos que no han sido utilizados para ajustar el modelo. En la medida en que el desempeño del modelo en ambos conjuntos de datos sea similar se puede decir que el modelo generaliza bien y, por tanto, queda validado por los datos disponibles.

Tanto el método de ajuste de modelos de gestión de tesorería basado en técnicas de Monte Carlo como la validación cruzada son métodos generales que permiten contemplar un elevado número de modelos. Este hecho facilita la comparación entre modelos de manera que puedan disiparse empíricamente las dudas sobre la utilización de un modelo u otro. Esta comparación debe realizarse no sólo entre modelos propuestos en la literatura sino también con respecto a modelos triviales basados en reglas sencillas. Este ejercicio garantiza que los esfuerzos realizados en la mejora de la toma de decisiones en la gestión de tesorería tienen sentido. 


\section{RESULTADOS}

Como resultado final de la metodología propuesta en este trabajo, el Gráfico 5 presenta un resumen visual de los pasos a seguir para la selección empírica de modelos de gestión de tesorería.

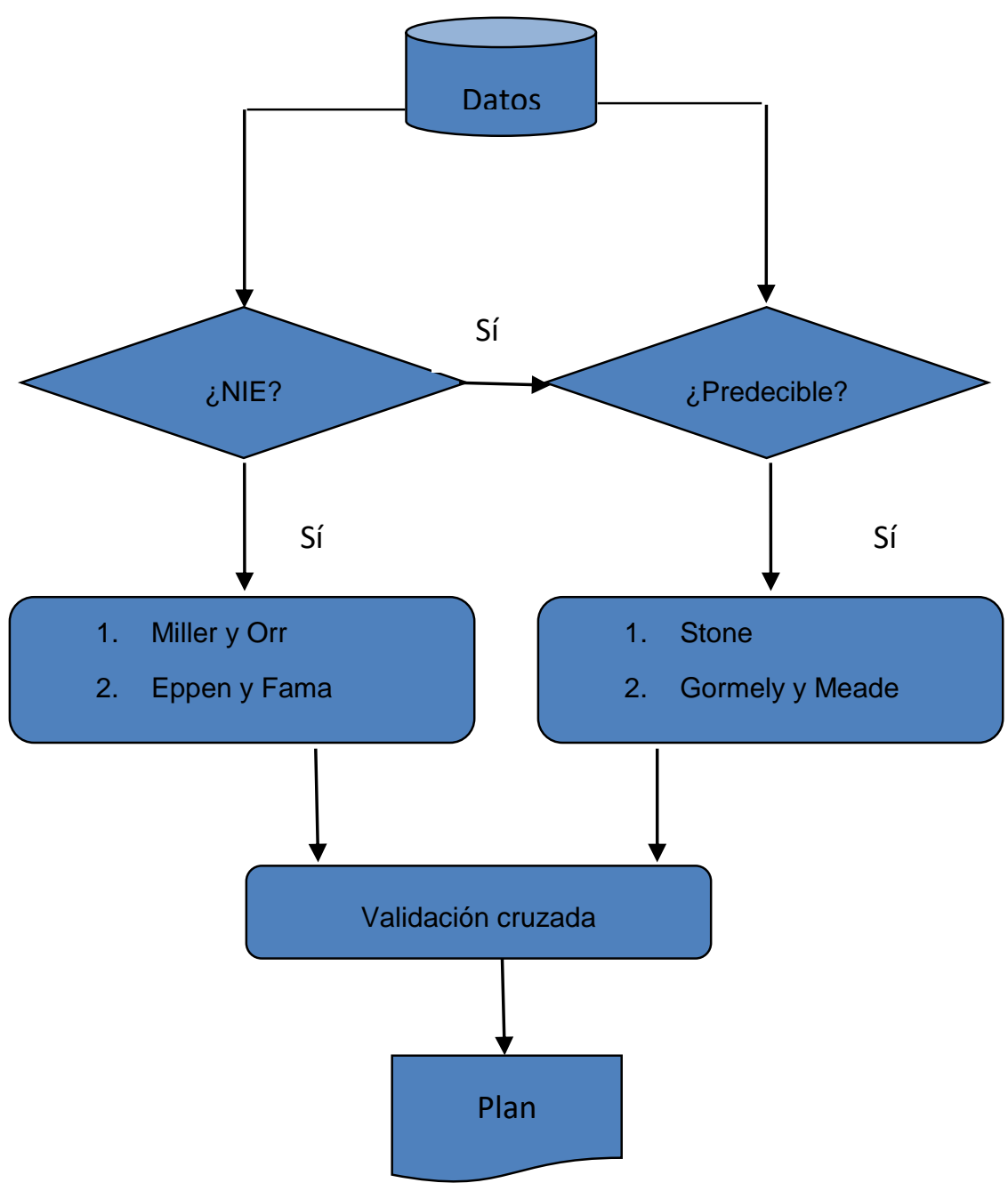

Gráfico 5. Selección empírica de modelos de gestión de tesorería.

Fuente: elaboración propia.

Como no puede ser de otra manera, la condición inicial para iniciar el proceso es la disponibilidad de una base de datos con los que trabajar. En este sentido, es importante destacar que no sólo es conveniente disponer de los flujos de caja sino de cualquier otro tipo de dato que pueda ser relevante para la extracción de patrones de comportamiento del flujo de caja. Además, si se pretende hacer una separación entre flujos de caja mayores y menores, será necesario disponer de los datos necesarios para la identificación de tales flujos.

Conviene recordar que la metodología descrita en el Gráfico 4 hace referencia a los flujos de caja menores ya que aquellos flujos de caja que se conocen con exactitud quedan fuera del 
análisis empírico. En la práctica, estos flujos de caja mayores serán considerados como datos de partida ciertos en la aplicación del modelo finalmente seleccionado.

El análisis empírico de los flujos de caja pretende responder dos preguntas cruciales para la selección del modelo. La primera es si se cumplen las hipótesis NIE. La segunda es si es posible obtener previsiones de los flujos caja que puedan utilizarse como entrada al modelo. La respuesta a estas cuestiones determinará el tipo de modelo que conviene elegir. Aunque ambas cuestiones no son incompatibles, obviamente la utilización de modelos que utilicen previsiones solo es posible si se utiliza algún modelo de previsión.

El paso previo a la utilización del modelo es la validación cruzada mediante la utilización de un training set y de un test set. Aunque por coherencia se omite en el Gráfico 4, en el caso de que la respuesta a las dos preguntas sea negativa no existe ninguna razón para descartar la posibilidad de realizar una validación cruzada de un modelo sin previsiones y evaluar su desempeño.

Finalmente, el modelo de gestión de tesorería que ha sido validado por los datos disponibles, puede empezar a utilizarse para planificar la toma de decisiones basadas en datos tal como se proponía en el Gráfico 1.

\section{CONCLUSIONES}

Parece evidente que la cantidad de datos disponible en las empresas para la toma de decisiones va a continuar creciendo de manera notable en los próximos años. Estos datos están ahí para quien los necesite, para quien quiera obtener un provecho de ellos. Desaprovechar este importante recurso para la toma de decisiones es equivalente a dejar el grifo de agua abierto malgastando un bien muy valioso.

En el ámbito de la gestión de tesorería, la utilización de modelos de gestión de tesorería puede contribuir a la mejora de la calidad de las decisiones financieras a corto plazo. La tesorería es el sistema circulatorio de la empresa y debe mantenerse en perfecto estado para evitar problemas indeseados. Sin embargo, la selección de uno de estos modelos no es una tarea sencilla. Para solucionar este problema, en este trabajo se ha propuesto una metodología empírica que partiendo de los datos disponibles en la empresa pretende seleccionar el modelo de gestión de tesorería más adecuado para cada empresa.

El análisis empírico de las características de los flujos de caja de una empresa constituye una primera contribución a la gestión de tesorería. Conocer qué tipo de componentes configuran el flujo de caja habitual de una empresa y qué propiedades estadísticas tienen ya aporta valor por sí mismo. Además, si la selección de modelos de gestión de tesorería está basada en este análisis empírico, la toma de decisiones estará mejor fundamentada.

Por último, el uso de técnicas de (big) data como las previsiones y la validación cruzada de modelos de gestión de tesorería son herramientas de gran utilidad para una toma de decisiones basada en datos en el ámbito de la gestión financiera a corto plazo. 


\section{REFERENCIAS BIBLIOGRÁFICAS}

da Costa Moraes, M. B., Nagano, M. S., \& Sobreiro, V. A. (2015). Stochastic cash flow management models: A literature review since the 1980s. Decision Models in Engineering and Management, 11-28.

Eppen, G. D., \& Fama, E. F. (1968). Solutions for cash-balance and simple dynamic-portfolio problems. The Journal of Business, 41(1), 94-112.

Gormley, F. M. and Meade, N. (2007). The utility of cash flow forecasts in the management of corporate cash balances. European journal of operational research, 182(2), 923-935.

Miller, M. H., \& Orr, D. (1966). A Model of the Demand for Money by Firms. The Quarterly journal of economics, 80(3), 413-435.

Pindado, J. and Vico, J. (1996). Evidencia empírica sobre los flujos de caja. Un nuevo enfoque en su tratamiento. Revista Española de Financiación y Contabilidad, 25(87), 497-517.

Ross, S. A., Westerfield, R., \& Jordan, B. D. (2002). Fundamentals of corporate finance (6th ed.). McGraw-Hill.

Salas-Molina, F., Pla-Santamaria, D., and Rodriguez-Aguilar, J. A. (2016). A multi-objective approach to the cash management problem. Annals of Operations Research. doi:10.1007/s10479-016-2359-1

Salas-Molina, F., Martin, F. J., Rodríguez-Aguilar, J. A., Serrà, J., and Arcos, J. L. (2017). Empowering cash managers to achieve cost savings by improving predictive accuracy. International Journal of Forecasting, 33(2), 403-415.

Stone, B. K. (1972). The use of forecasts and smoothing in control-limit models for cash management. Financial Management, 1(1), 72-84. 